Invited review to appear in the proceedings of the Warner Symposium on Cataclysmic Variables, New Astronomy Reviews, 1999, eds. P.A. Charles, A.R. King \& D. O'Donoghue

\title{
Secondary Stars in CVs: The Theoretical Perspective
}

\author{
Ulrich Kolb ${ }^{\mathrm{a}}$ and Isabelle Baraffe ${ }^{\mathrm{b}}$ \\ ${ }^{a}$ Dept. of Physics 83 Astronomy, University of Leicester, Leicester LE1 7RH, UK \\ ${ }^{\mathrm{b}}$ Ecole Normale Supérieure de Lyon, \\ C.R.A.L. (UMR 5574 CNRS), F-69364 Lyon Cedex 07, France
}

\begin{abstract}
We apply the new generation of theoretical models of low-mass stars to secondaries in CVs, focussing on systems above the period gap. The models confirm that the spectral type should be a good indicator of the donor mass. The orbital period-spectral type diagram can potentially constrain the long-term mean mass transfer rate. A transfer rate that increases with decreasing period is most easily reconciled with the observational data.
\end{abstract}

\section{Introduction}

The new generation of low-mass star and brown dwarf models by Baraffe et al. (1995, 1997, 1998, henceforth summarized as BCAH) represent a significant improvement in the quantitative description of stars with mass $\lesssim 1 \mathrm{M}_{\odot}$.

The main strengths of the models are in two areas: the microphysics determining the equation of state (EOS) in the stellar interior, and the realistic non-grey atmosphere models which enter as the outer boundary condition. The EOS (Saumon, Chabrier and Van Horn 1995) is specifically calculated for very low-mass stars, brown dwarfs and giant planets. It has been successfully tested against recent laserdriven shock wave experiments performed at Livermore, which probe the complex regime of pressure dissociation and ionization relevant for these objects (cf. Saumon et al. 1998). Recent much improved cool atmosphere models (see e.g. the review of Allard et al. 1997) now provide realistic atmosphere profiles, which we use as the outer boundary condition, and synthetic spectra. Chabrier \& Baraffe (1997) have shown that evolutionary models employing a grey atmosphere instead, e.g. the standard Eddington approximation, overestimate the effective temperature for a given mass, and yield too large a minimum hydrogen burning mass.

Several observational tests confirm the success of evolutionary models based on these improvements, e.g. mass-magnitude relations, colour-magnitude diagrams (Baraffe et al. 1997, 1998), mass-spectral type relations (Baraffe \& Chabrier 1996), the first cool BD GL 229B (Allard et al. 1996), properties of components in detached eclipsing binaries (Chabrier \& Baraffe 1995) and of field M-dwarfs (cf. Beuermann et al. 1998).

Here we consider the BCAH models in the context of CV secondaries, and focus on the relation between spectral type $(S p T)$, donor mass $\left(M_{2}\right)$ and orbital period $(P)$. We obtain the spectral type of a stellar model from its calculated colour $(I-K)$ and the empirical $S p T-(I-K)$ relation established by Beuermann et al. (1998). A summary of the input physics used for the calculations presented below is given by Kolb \& Baraffe (1999).

\footnotetext{
${ }^{1}$ Present address: Dept. of Physics, The Open University, Walton Hall, Milton Keynes MK7 6AA
} 


\section{The mass-spectral type relation}

Mass loss causes stars to deviate from thermal equilibrium. The surface luminosity is no longer in balance with the luminosity generated in the core by nuclear reactions, and the difference causes the star to expand or contract. Therefore the stellar radius can be either larger or smaller than the corresponding equilibrium radius (see e.g. Whyte \& Eggleton 1980). Remarkably, the effective temperature of lowmass main-sequence stars is rather insensitive to the degree of thermal disequilibrium. They behave just like giant stars on the Hayashi line, expanding along an evolutionary track with nearly constant effective temperature. This can be understood in terms of simple homology relations for predominantly convective low-mass stars (e.g Stehle et al. 1996).

Using BCAH models we quantify the deviation from equilibrium spectral type, as a measure of the surface temperature, for $\mathrm{CV}$ donors with various evolutionary histories. We consider the following simple cases:

Standard sequence: mass transfer starts from an unevolved (ZAMS) donor with mass $1 \mathrm{M}_{\odot}$, proceeds at a constant rate $1.5 \times 10^{-9} \mathrm{M}_{\odot} \mathrm{yr}^{-1}$, stops when the donor becomes fully convective (at mass 0.21 $\mathrm{M}_{\odot}$ ), and resumes at the lower rate $5 \times 10^{-11} \mathrm{M}_{\odot} \mathrm{yr}^{-1}$ once the donor has settled back into thermal equlibrium. This sequence fits the period gap in the framework of disrupted orbital braking (e.g. King 1988, Kolb 1996).

Unevolved sequences with constant $\dot{M}$ : mass transfer starts from an unevolved (ZAMS) donor with mass $1 \mathrm{M}_{\odot}$ and proceeds at a constant rate $\dot{M}\left(10^{-8} \mathrm{M}_{\odot} \mathrm{yr}^{-1}\right.$ or $\left.10^{-7} \mathrm{M}_{\odot} \mathrm{yr}^{-1}\right)$.

Evolved sequences with constant $\dot{M}$ : mass transfer initiates from a donor which has already burned a significant fraction of its hydrogen supply, but is still in the core hydrogen burning phase. We show three examples: a moderately evolved low $\dot{M}$ sequence (initial central hydrogen content $X_{c}=0.16$, initial donor mass $M_{2}=1.0 \mathrm{M}_{\odot}, \dot{M}=1.5 \times 10^{-9} \mathrm{M}_{\odot} \mathrm{yr}^{-1}$ ), an evolved, low $\dot{M}$ sequence $\left(X_{c}=4 \times 10^{-4}, M_{2}=1.2 \mathrm{M}_{\odot}, \dot{M}=1.5 \times 10^{-9} \mathrm{M}_{\odot} \mathrm{yr}^{-1}\right)$, and an evolved high $\dot{M}$ sequence $\left(X_{c}=4 \times 10^{-4}, M_{2}=1.0 \mathrm{M}_{\odot}, \dot{M}=5 \times 10^{-8} \mathrm{M}_{\odot} \mathrm{yr}^{-1}\right)$.

Note that as the mass loss timescale is short compared with the nuclear time, the nuclear state of the donor is essentially frozen once mass transfer has started.

Fig. 11 shows that the effect of thermal disequilibrium is negligible along the standard sequence; this hardly differs from that for CVs with a ZAMS donor. If $\dot{M}$ is higher than in the standard sequence, the spectral type is slightly later for a given mass, while in a sequence with evolved donors it is somewhat earlier. As most CVs should form with essentially unevolved donors (Politano 1996, de Kool 1992), and as the estimated $\dot{M}$ exceeds $10^{-8} \mathrm{M}_{\odot} \mathrm{yr}^{-1}$ only for very few CVs (e.g. Warner 1995, his Fig. 9.8), the upper solid curve $\left(X_{c}=0.16\right)$ and the dashed curve $\left(M=10^{-8} \mathrm{M}_{\odot} \mathrm{yr}^{-1}\right)$ in Fig. 11 should bracket the location of the majority of CVs in the mass-spectral type diagram. The figure suggests that the spectral type is a good indicator of the donor mass, with a typical uncertainty $\Delta M_{2} \simeq 0.1 \mathrm{M}_{\odot}$, for any given $S p T$ earlier than M0-M2. For later SpT this mass "determination" becomes impractical as the curves in the figure flatten and $\Delta M_{2}$ is large. Even with extreme evolutionary cases, the unevolved sequence with very high transfer rate $\left(\dot{M}=10^{-7} \mathrm{M}_{\odot} \mathrm{yr}^{-1}\right)$ more typical for supersoft $\mathrm{X}$-ray binaries than for $\mathrm{CVs}$, and the evolved sequence starting on the terminal main-sequence, the range covered by the secondary stars in the $S p T$-mass diagram remains surprisingly narrow (Fig. 2; see Kolb, King \& Baraffe 1999 for more details).

Although the observations compiled by Smith \& Dhillon (1998) do not seem to support this prediction, the observational errors on $M_{2}$ are far too large for any definitive conclusions to be drawn from this mismatch. On the contrary, with improved and more reliable mass determinations, one may hope to constrain the range of $\dot{M}$ and the relative importance of evolved and unevolved systems in the CV population. 


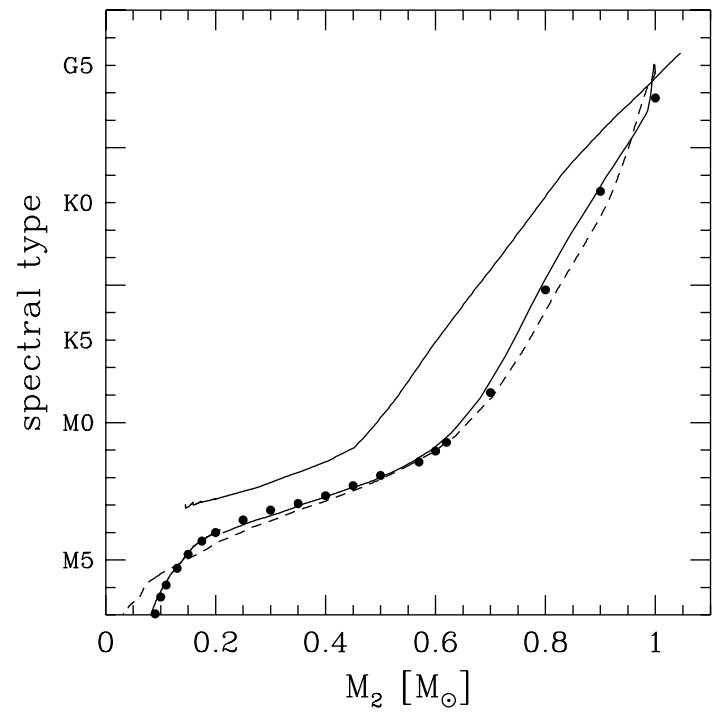

Fig. 1. Spectral type versus secondary mass. Dots denote ZAMS models. Solid curve, close to the dots: standard sequence; solid curve, above the dots: donor moderately evolved $\left(X_{c}=0.16\right)$. Dashed: donor unevolved, high $\dot{M}\left(10^{-8} \mathrm{M}_{\odot} \mathrm{yr}^{-1}\right)$.

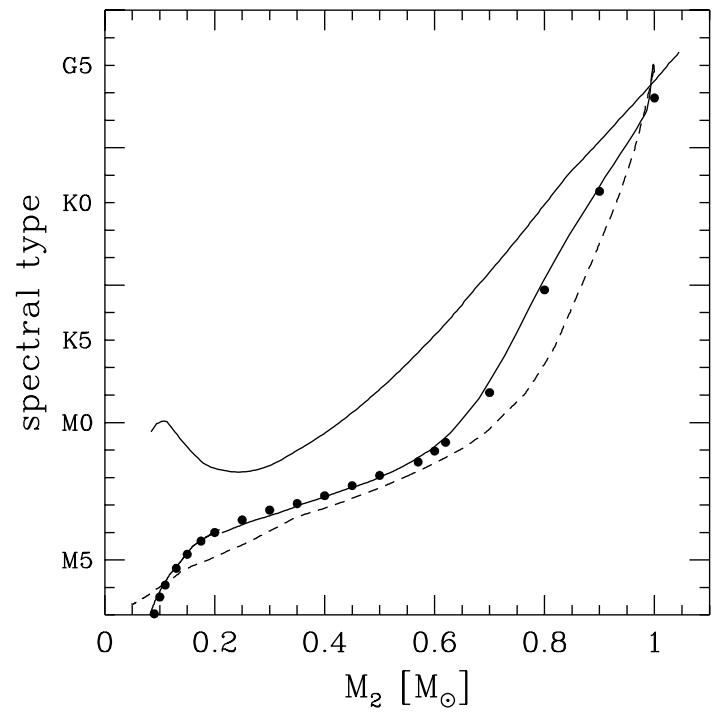

Fig. 2. Same as Fig. 1, but with extreme assumptions for the evolved sequence (low $\dot{M}, X_{c}=4 \times 10^{-4}$ ) and the unevolved high $\dot{M}$ sequence $\left(10^{-7} \mathrm{M}_{\odot} \mathrm{yr}^{-1}\right)$.

The figures also apply to secondaries in LMXBs. Evolutionary considerations suggest that, unlike CVs, most short-period LMXBs, form with an evolved main-sequence donor with mass $\gtrsim 1 \mathrm{M}_{\odot}$ (King \& Kolb 1997, Kalogera et al. 1998).

\section{The orbital period-spectral type diagram}

A second invaluable diagnostic diagram for CVs is the orbital period-spectral type diagram (PS diagram) - the "HR diagram" analogue for CV secondary stars. In a semi-detached binary the Roche lobe filling star's mean density $\rho$ determines the orbital period $P$ almost uniquely (e.g. King 1988), $P_{h}=k / \rho_{\odot}^{1 / 2}$, with $k \simeq 8.85$ being only a weak function of the mass ratio, $P_{h}$ the period in hr, and $\rho_{\odot}$

the mean density in solar units. Hence the PS diagram probes the donor structure in terms of two stellar parameters that are relatively easy to determine from observation. (The importance of the PS diagram has already been pointed out by Ritter 1994).

The ZAMS track defines an upper bound for the scatter of observed CV secondaries in Fig. 3. Systems immediately above and below the period gap have the same spectral type. The results above suggest that these CVs have the same donor mass, consistent with the standard period gap model according to which CVs evolve through the gap with constant secondary mass.

The location of various evolutionary tracks above the period gap are shown in Fig. 5. Most tracks intersect the ZAMS track at a critical period $P_{\text {crit }} \simeq 5-6 \mathrm{hr}$, which corresponds to a donor with mass $M_{2} \simeq 0.6 \mathrm{M}_{\odot}$ marking the transition from mainly radiative to predominantly convective stars. A simple 


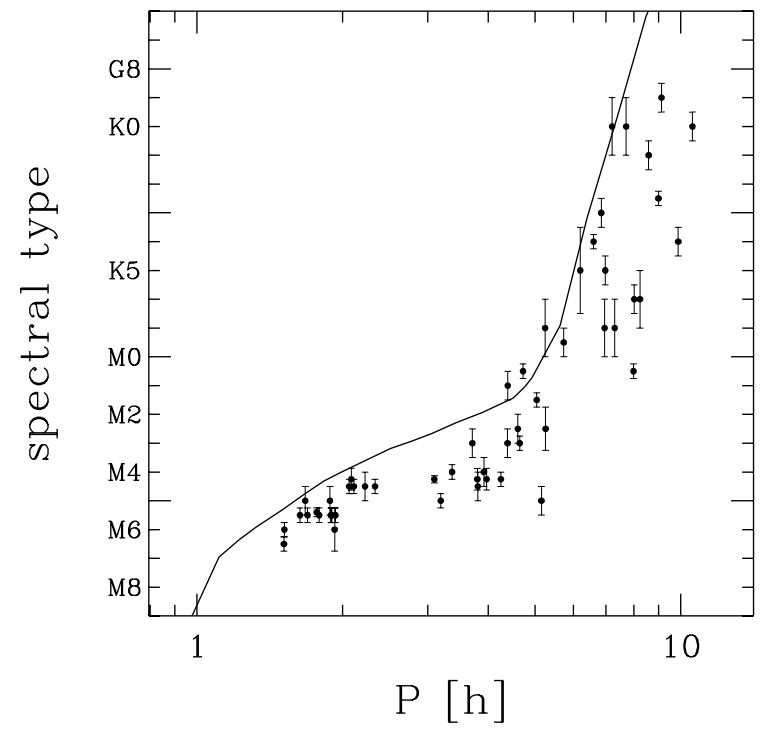

Fig. 3. Spectral type versus orbital period for CV secondaries. Data taken from Beuermann et al. 1998. Solid: theoretical location of ZAMS secondaries.

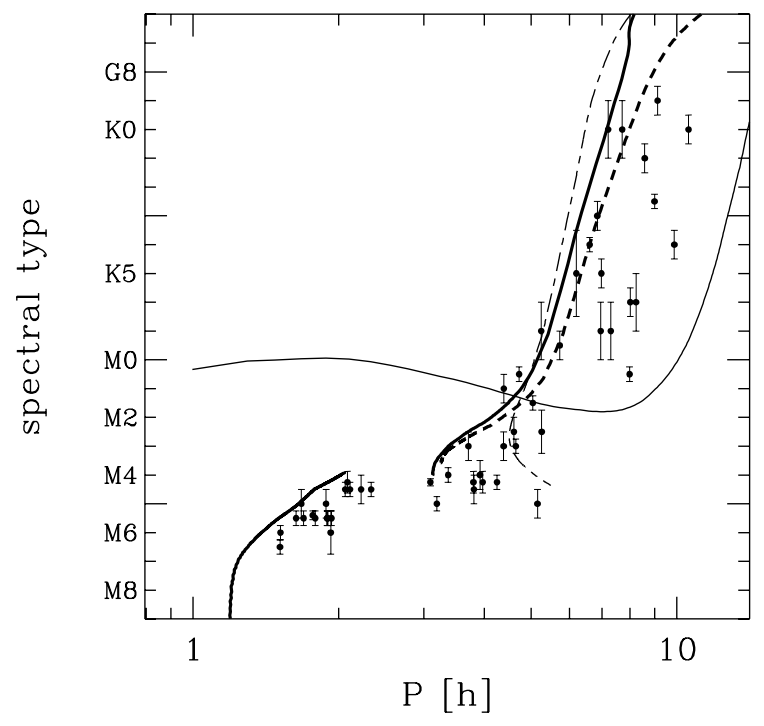

Fig. 4. Same as Fig 3, but with various evolutionary sequences, cf. also the track morphology in Fig. 5 . Thick solid: standard sequence; thin solid: evolved low $\dot{M}$ sequence $\left(X_{c}=4 \times 10^{-4}\right)$; short/long dashes: unevolved sequence $\left(\dot{M}=10^{-8} \mathrm{M}_{\odot} \mathrm{yr}^{-1}\right)$; thick dashes: evolved high $\dot{M}$ sequence $\left(X_{c}=4 \times 10^{-4}\right)$.

corollary from the results of Sec. 2 is that the donor mass on any evolutionary track is about the same as the one on the ZAMS track with the same spectral type. Deviations to shorter or longer periods from the ZAMS track mean that the donor is smaller or larger then the corresponding ZAMS star, respectively. From the morphology of tracks in Fig. 5 it is clear that unevolved high $\dot{M}$ sequences are excluded at long periods $\left(P \gtrsim P_{\text {crit }}\right.$ ), but would explain the observed scatter for $P \lesssim P_{\text {crit. }}$. Likewise, the observations exclude evolved low $\dot{M}$ sequences at short periods, while these are clearly required for $P \gtrsim P_{\text {crit }}$.

This suggests that, overall, a secular mean mass transfer rate increasing with decreasing orbital period would provide a good fit to the observed PS diagram above the period gap. CVs would evolve along a low $\dot{M}$ track at long periods and along a high $\dot{M}$ track at short periods. To explain the scatter of systems at long periods the full spectrum of nuclear evolution prior to mass loss is required. Note that the sequence starting mass transfer from a star at the terminal main-sequence defines a lower bound for the data points in Fig. 囵. It is difficult to see why CVs with somewhat evolved donors should dominate the population. One possibility is that unevolved systems are more susceptible to irradiation-driven mass transfer cycles (King et al. 1996), with a high state $\dot{M}$ too high to be recognisable as CVs. The scatter at short periods $(3-5 \mathrm{hr})$ in turn seems to imply a wide range of secular mean $\dot{M}$ values, $\left(10^{-9}-10^{-8}\right.$ $\left.\mathrm{M}_{\odot} \mathrm{yr}^{-1}\right)$. None of the orbital angular momentum loss mechanisms postulated so far generates this wide a range of $\dot{M}$ at a given $P$, while observational estimates for $\dot{M}$ always suggest that there is an even larger range (e.g. Patterson 1984, Warner 1995). 


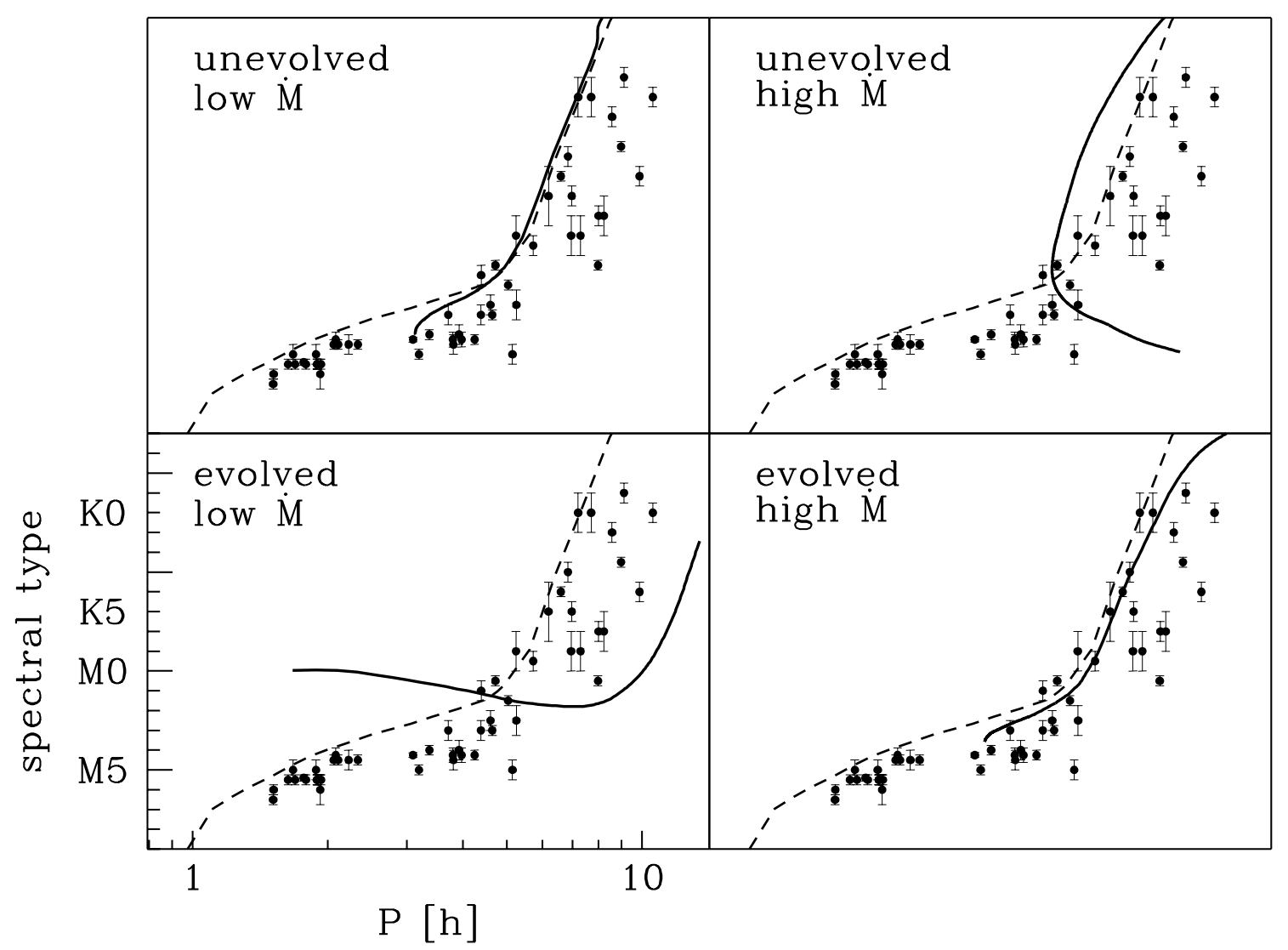

Fig. 5. Morphology of evolutionary tracks in the spectral type--orbital period diagram. Dashed: location of ZAMS secondaries. Solid curves: standard sequence (upper left panel), unevolved sequence with $\dot{M}=10^{-7} \mathrm{M}_{\odot} \mathrm{yr}^{-1}$ (upper right), evolved sequence $\left(X_{c}=4 \times 10^{-4}\right)$ with $\dot{M}=1.5 \times 10^{-9} \mathrm{M}_{\odot} \mathrm{yr}^{-1}$ (lower left), and with $\dot{M}=5 \times 10^{-8}$ $\mathrm{M}_{\odot} \mathrm{yr}^{-1}$ (lower right).

If the $\dot{M}$ range reflects the range of the secular mean, then the appearance of a gap with relatively sharp edges is more difficult to understand than with a uniform evolution driven by angular momentum losses insensitive to system parameters (Kolb 1996; Stehle et al. 1996). Yet the observed period distribution and its explanation by disrupted orbital braking cannot unambiguously rule out a large range of the secular mean transfer rate above the gap (Baraffe \& Kolb 1999). The $\dot{M}$ range certainly has to level off rather steeply below $10^{-9} \mathrm{M}_{\odot} \mathrm{yr}^{-1}$ to avoid filling the gap with systems that hardly detach. On the other hand, systems with $\dot{M} \gtrsim 2 \times 10^{-9} \mathrm{M}_{\odot} \mathrm{yr}^{-1}$ undergo period bounce before they reach the upper edge of the gap, and before the donor becomes fully convective (at a mass $M_{\text {conv }}$ ). This bounce period increases with $\dot{M}$, while $M_{\text {conv }}$ decreases. Hence the detached phase of high $\dot{M}$ systems extends from periods longer than $3 \mathrm{hr}$ to periods shorter than $2 \mathrm{hr}$, and does not interfere with the "classical" gap. The time spent in the detached phase increases as well, in the case $\dot{M}=10^{-8} \mathrm{M}_{\odot} \mathrm{yr}^{-1}$ it is longer than 8 Gyr (assuming 
angular momentum losses by gravitational radiation). Only a small fraction of such systems would reach the orbital period minimum at 80 min within the age of the Galaxy, thus alleviating (but not solving) the problem of the missing period spike (see Kolb \& Baraffe 1999) at this minimum period.

Our conclusions from the mass-spectral type diagram and orbital period-spectral type diagram can be tested by a set of more accurate mass and radius determinations of CV secondaries.

\section{References}

Allard, F. 1999, in Very Low-Mass Stars and Brown Dwarfs in Stellar Clusters and Associations, Euroconference, La Palma 1998

Allard, F., Hauschildt, P.H., Baraffe, I., Chabrier, G. 1996, APJ, 465, L123

Allard F., Hauschildt P.H., Alexander D.R., Starrfield S. 1997, ARA\&A, 35, 137

Baraffe I., Chabrier G. 1996, ApJ, 461, L51

Baraffe I., Kolb U. 1999, MNRAS, submitted

Baraffe I., Chabrier G., Allard F., Hauschildt P.H. 1995, ApJ, 446, L35

Baraffe I., Chabrier G., Allard F., Hauschildt P.H. 1997, A\&A, 327, 1054

Baraffe I., Chabrier G., Allard F., Hauschildt P.H. 1998, A\&A, 337, 403

Beuermann, K., Baraffe, I., Kolb, U., \& Weichhold, M. 1998, A\&A, 339, 518

Chabrier G., Baraffe I. 1995, ApJ, 451, L29

Chabrier G., Baraffe I. 1997, A\&A, 327, 1039

Kalogera V., Kolb U., King A.R. 1998, ApJ, 504, 967

King A.R. 1988, QJRAS, 29, 1

King A.R., Kolb U. 1997, ApJ, 481, 918

King A.R., Frank J., Kolb U., Ritter H. 1996, ApJ, 467, 761

Kolb U. 1996, in Cataclysmic Variables and Related Objects, ed. A. Evans, J.H. Wood, Dordrecht: Kluwer, IAU Coll. 158, 433

Kolb U., Baraffe I. 1999, MNRAS, submitted

Kolb U., Ritter H. 1992, A\&A, 254, 213

Kolb U., King A.R., Baraffe I., 1999, MNRAS, submitted

de Kool M. 1992, A\&A, 261, 188

Mazzitelli I. 1989, ApJ, 340, 249

Patterson J. 1984, ApJS, 54, 443

Politano M. 1996, ApJ, 465, 338

Ritter H., 1994, in Evolutionary Links in the Zoo of Interactive Binaries, ed. F. D'Antona, V. Caloi, C. Maceroni,

F. Giovanelli, in Memorie della Società Astronomica Italiana 65, 173

Saumon, D., Chabrier, G., and VanHorn, H.M., 1995, ApJS, 99, 713

Saumon, D., Chabrier, G., Wagner, D.J., Xie, X., 1998, Phys. Rev., submitted

Smith D.A., Dhillon V.S. 1998, MNRAS, 301, 767

Stehle R., Ritter H., Kolb U. 1996, MNRAS, 279, 581

Warner B. 1995, Cataclysmic Variable Stars, Cambridge Astrophysics Series 28, Cambridge: CUP

Whyte C., Eggleton P.P. 1980, MNRAS, 190, 801 